\title{
Traffic aware cell selection algorithm for Tetra Trunk based professional mobile radio
}

\author{
Berna Özbek $^{1}$ (D) $\cdot$ Azad Karataş ${ }^{1} \cdot$ Erinç Deniz Bardak $^{2}$. Ilker Sönmez ${ }^{2}$
}

Published online: 3 January 2019

๑) Springer Science+Business Media, LLC, part of Springer Nature 2019

\begin{abstract}
Load balancing and traffic management are the critical needs in cell selection decision for a better and seamless communication demands in professional mobile radios. For the cases where cell selection algorithms do not consider the traffic load, there may be call drops due to the congestion in networks or longer call setup times for the users. These undesired consequences can cause dramatic quality degradation especially for the emergency cases or public safety services. In this paper, we propose two algorithms for Tetra Trunk based professional mobile radios by considering both traffic load and received signal strength indication (RSSI) in order to reduce the significant delays while establishing transmissions. The proposed full set cell selection algorithm is prioritized to reduce the call setup time for the mobile users and the proposed reduced set cell selection algorithm is focused on minimizing the number of RSSI measurements which causes significant delay in practical professional mobile radio. We illustrate the performance results in terms of delay for Tetra Trunk based professional mobile radio.
\end{abstract}

Keywords Tetra Trunk $\cdot$ Professional mobile radio $\cdot$ Cell selection algorithm $\cdot$ Traffic aware

\section{Introduction}

Recently, the demand for Professional Mobile Radio (PMR) has been dramatically increased for public safety network in wireless communications. This causes the need for higher bandwidth efficient systems and traffic management. Terrestrial Trunked Radio (Tetra) is one of the promising solutions and bandwidth efficient standard published by European Telecommunication Standards Institute (ETSI) in 1995.

TETRA is a technical platform with data and voice services and targeted for the needs of emergency services, government agencies, military and transport services. Tetra base stations (BSs) support antenna diversity techniques such

\footnotetext{
Berna Özbek

bernaozbek@iyte.edu.tr

Azad Karataş

azadkaratas@iyte.edu.tr

Erinç Deniz Bardak

edbardak@aselsan.com.tr

İlker Sönmez

isonmez@aselsan.com.tr

1 Department of Electrical and Electronics Engineering, İzmir Institute of Technology, Izmir, Turkey

2 ASELSAN A.Ş., Ankara, Turkey
}

as selection diversity, equal gain combining and maximal ratio combining. The studies have shown that [1] selection diversity improves the Bit Error Rate (BER) significantly for Tetra systems. Due to the limitations of available bands, efficient cell selection algorithms are required to guarantee the demands for PMR systems, as seamless connectivity and fast call setup time.

In the literature, there are several efficient cell selection algorithms for the cellular systems. In order to distribute the traffic load in mobile systems fairly, mobility load balancing has been presented in [2] to improve the efficiency of resource utilization. In [3], traffic load scheme has been examined while maximizing the throughput of traffic control. while assigning the users to the cell with minimum load to minimize the call blocking ratio. In [4], the congested network load has been transfered to less congested network by adapting transmitter power level of users and switching them between cell sites. In [5], a coalitional game has been formulated for joint cell selection and resource allocation problem. The cell selection algorithm in [6] has been balanced the load to offload the excess traffics based on n-dimensional Markov chain.

In [7], cell selection process has been applied with a competition based among group of users. Each user in groups selects the cell with the minimum required transmit power. 
The results of this approach gives better performance in terms of number of supportable users and transmit power levels for a target SINR value. In [8], a cell selection algorithm has been presented to improve the system energy efficiency of small cell networks by considering both user equipment and backhaul link data rate constraints. In [9], the cell selection algorithm has considered both load balancing and total energy consumption. The users are assigned to the femtocells considering the capacity improvement obtained per unit increase in transmit power. In [10], a cell selection problem has been formulated as hidden Markov model by creating a candidate set and targeting handover base station (BS) to observe and predict cell load information of BSs.

The cell selection algorithms for the cellular networks have not been applied directly to Tetra Trunk based PMR systems. In this paper, we propose two cell selection algorithms by taking into account both traffic load and received signal strength indication (RSSI) values. The goal of the proposed traffic aware full set cell selection algorithm is to reduce call setup while the objective of the proposed traffic aware reduced set cell selection algorithm is to minimize also the number of RSSI measurements. Compared to the our previous works in [11] in which the cell load has been calculated by using the number of connected users, in this work, we determine the traffic load based on the call duration in a realistic Tetra Trunk scenario including a cell re-selection procedure.

The rest of the paper is organized as follows. In Sect. 2, system model is given. In Sects. 3 and 4, the proposed traffic aware full set and reduced set cell selection algorithms are described in detail. Then, the performance evaluations are provided in Sect. 5 and the work is concluded in Sect. 6.

\section{System model}

Time Division Multiple Access technique in Tetra Trunk standard provides four user channels on one radio carrier and $25 \mathrm{kHz}$ spacing between carriers by using $\pi / 4$ DQPSK modulation scheme [12]. Each BS $u$ has a total number of timeslots $M_{u}$ :

$M_{u}=\frac{4 B}{\Delta f}$

where $B$ represents the bandwidth and $\Delta f$ is the channel spacing. One of these time-slots is used for control and the users can have several time slots to obtain higher transmission capacity.
Each user $k$ measures instantaneous RSSI value for the BS $u$ as follows:

$$
\begin{aligned}
R S S I_{u, k}(d B)= & E I R P_{u}-P L_{u, k}-B u L-S h_{u, k}-B L \\
& +G_{r}-C L_{r}-F_{u, k}
\end{aligned}
$$

where $P L_{u, k}$ is path loss between user $k$ and the BS $u, B u L$ is building loss, $S h_{u, k}$ is log-normal shadowing, $B L$ is body loss, $G_{r}$ is receiver antenna gain, $C L_{r}$ is receiver cable loss and $F_{u, k}$ is fading coefficient. $E I R P_{u}$ is effective isotropic radiated power value for the $\mathrm{BS} u$ :

$E I R P_{u}(d B)=P_{t}+G_{t}-C L_{t}$

where $P_{t}$ is transmit power, $G_{t}$ is transmitter antenna gain and $C L_{t}$ is transmitter antenna cable loss.

In $\mathrm{C} 1$ based cell selection algorithm [13], all users firstly calculate the loss parameter for the BS $u$ [12]:

$$
\begin{aligned}
C 1_{u, k}= & P r_{u, k}-R e c \_S e n s \\
& -\max \left(0, M s_{-} T x P w r_{-} M a x_{-} C e l l-P_{M S M A X}\right)
\end{aligned}
$$

where $P r_{u, k}$ is the received power of user $k$ from the BS $u$ obtained by averaging $N_{s}$ consecutive RSSI values, Rec_Sens denotes minimum acceptable received power at the mobile user, Ms_TxPwr_Max_Cell stands for the maximum allowable transmit power at that channel and $P_{M S M A X}$ is the maximum transmit power for $\pi / 4$ DQPSK modulation.

After $\mathrm{C} 1$ path loss calculation, each user adds the BS that has $\mathrm{C} 1$ higher than 0 to their candidate sets. These cells are sorted in descending order and users attempt to attach to the first BS with available capacity. The BS is called available if it has enough time slots for the user to be attached.

\section{Proposed traffic aware full set cell selection}

In the proposed traffic aware full set cell selection algorithm, the system focuses on the distribution of users over the BSs which affects directly the system performance in terms of delay. Therefore, the proposed full set algorithm is not take into account the total number of RSSI measurements while establishing transmission link. The procedure of the proposed full set algorithm is given in the following:

1. Constructing the set based on receiver sensitivity:

Each user $k$ measures the RSSI values from all BSs and then add the index of BS into set $\mathbb{P}_{k}$ satisfying the criterion given below.

$$
\mathbb{P}_{k}=\left\{u \in N_{b}: R S S I_{u, k} \geq R S S I_{t h}\right\}
$$


where $N_{b}$ is the total number of BSs in the considered system and $R S S I_{t h}$ is the receiver sensitivity.

2. Calculating the utility value:

For user $k$, utility value is calculated by considering the RSSI values and traffic load information.

At each BS, the unmapped traffic load (UTL) information is determined by,

$U T L_{u}=(1-c) \frac{I_{u}}{N_{u} / N_{b}}+c \frac{1}{M_{u}} \frac{H}{D}$

where $I_{u}$ is the number of inactive users which can not establish a call yet, $N_{u}$ is the total number of users $H$ represents the duration of talks of active users in seconds, $D$ stands for the defined time interval and $c$ is the weight for the active users. BSs include the total traffic volume of defined time interval in their cell broadcast information. Each BS broadcasts the index of $U T L_{u}$ obtained from the predefined table given in the next sections.

In Tetra Trunk systems, all users measures RSSI values and obtain the UTL information from all BSs in a given time interval. Then, the user $k$ calculates the utility values for all BSs:

$U_{u, k}=w f\left(R S S I_{u, k}\right)+(1-w) h\left(U T L_{u}\right), \quad \forall u \in \mathbb{P}_{k}$

where $w$ is the weight of either RSSI or UTL value, the function $f($.$) is mapped the measured RSSI to the sorted$ RSSI, the function $h($.) transforms the UTL value to the mapped traffic load value. These mapping functions will be given in the next sections.

For initial cell selection, BSs broadcast a traffic load information by considering only the number of inactive users attached to the BSs. Therefore, for the initial UTL calculation, $c$ parameter is set to 0 since there will be no active users and no calls occurred.

For cell re-selections, in addition to the inactive users term, UTL value includes a term keeping history of talking time duration of active users for the last defined traffic duration.

3. Attaching to the BS:

In order to reduce the delay, the user $k$ sends a request for attaching to the BS with the highest utility function as:

$$
u_{k}^{*}=\underset{\forall u \in \mathbb{P}_{k}}{\operatorname{argmax}} U_{u, k}
$$

The flowchart of the traffic aware full set cell selection algorithm for each user $k$ is given in Fig. 1 .

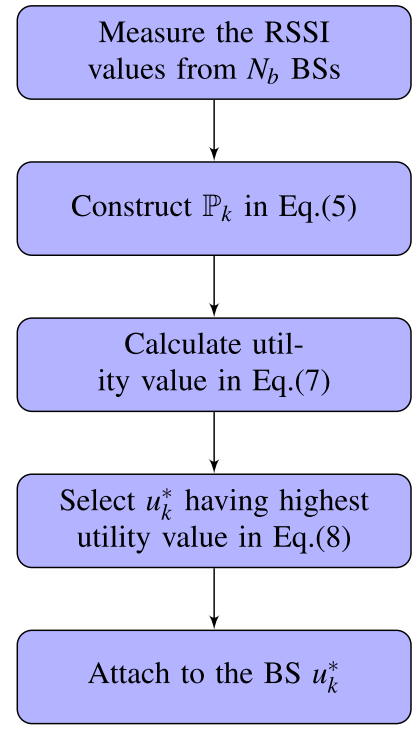

Fig. 1 Flowchart of the proposed traffic aware full set cell selection algorithm

\section{Proposed traffic aware reduced set cell selection}

The proposed traffic aware reduced set cell selection algorithm considers both the traffic load in the cells while shortening the elapsed time to attach to a BS. One of the main causes of this delay is to obtain RSSI measurements from all BSs. Since the proposed reduced set algorithm intends to decrease the elapsed time by reducing the RSSI measurements unlike the proposed full set algorithm. While reducing the connection time, the proposed reduced set algorithm also takes into account the traffic load by assigning the users to the BSs fairly. The unbalanced distribution of users among cells may cause higher waiting time to communicate because of the limited number of available channels in Tetra Trunk. The proposed traffic aware reduced set cell selection is described as follows:

1. Constructing the neighbour cells set:

With the aid of global positioning system (GPS), the users create their set of neighbour cells, $\mathbb{Z}_{k}$ and sort them in ascending order. Then, all users measure the RSSI values of the BSs starting from the nearest one.

2. Calculating the utility value:

All users calculate their utility values starting from the first BS in the set $\mathbb{Z}_{k}$ as:

$U_{r, k}^{\prime}=w g\left(R S S I_{r, k}\right)+(1-w) h\left(U T L_{r}\right) \quad \forall r \in \mathbb{Z}_{k}$

where the function $g($.$) is mapped the measured RSSI$ value to the normalized RSSI. This mapping function will be described in the next sections. 
Fig. 2 Flowchart of the proposed reduced set cell selection algorithm

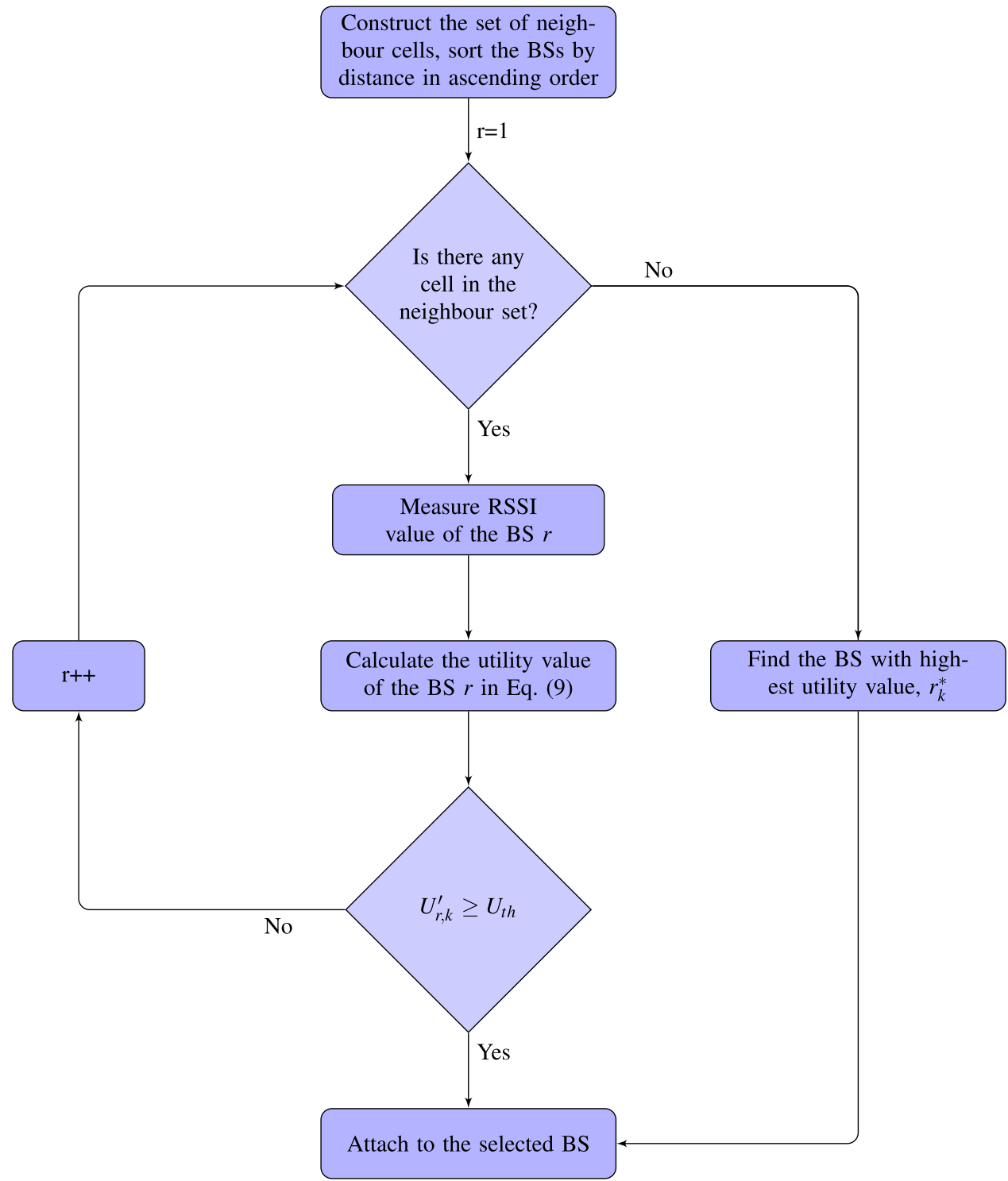

3. Attaching to the BS:

If the corresponding utility value is higher than a defined utility threshold value, $U_{t h}$, the user attaches to this BS. If this condition is not satisfied by this BS, the user connects the next cell in its neighbor set one by one. If there are no cells that satisfies this utility threshold value, then user attaches to the BS having the highest utility value.

The flowchart of the proposed traffic aware reduced set cell selection algorithm is given for each user $k$ in Fig. 2.

\section{Performance evaluations}

In order to implement the practical Tetra Trunk PMR scenario, the users are divided into a number of groups. The group calls can only occur between the same group in which
Table 1 Users with mobility information

\begin{tabular}{ll}
\hline User type & Velocity \\
\hline Immobile & $0 \mathrm{~km} / \mathrm{h}$ \\
Pedestrian & Up to $3 \mathrm{~km} / \mathrm{h}$ \\
Vehicle & Up to $80 \mathrm{~km} / \mathrm{h}$ \\
\hline
\end{tabular}

the users can be both inactive and active during simulation time. All talks in group conversations are arranged by considering short talk duration within 5 and $15 \mathrm{~s}$. The number of active users in the groups is determined with the probability of push-to-talk (PTT). These group talks are broadcasted by BSs to all users in the group so that each listener user does not need for an assigned channel. Channels are occupied by active users who establish a talk. If the serving cell has no available channel for the active user, then the user is queued by this BS. Therefore, this user must wait until there 
Table 2 Sorted RSSI values in $f$ function for the full set

\begin{tabular}{llcl}
\hline Sorted RSSI indices & $\mathrm{f}()$. & Sorted RSSI indices & $\mathrm{f}()$. \\
\hline 1 & 1 & 8 & 0.5 \\
2 & 0.928 & 9 & 0.429 \\
3 & 0.857 & 10 & 0.357 \\
4 & 0.786 & 11 & 0.286 \\
5 & 0.714 & 12 & 0.214 \\
6 & 0.643 & 13 & 0.143 \\
7 & 0.571 & 14 & 0.071 \\
\hline
\end{tabular}

Table 3 Normalized RSSI values in $g$ function for the reduced set

\begin{tabular}{llll}
\hline RSSI value $(\mathrm{dBm})$ & $\mathrm{g}()$. & RSSI value $(\mathrm{dBm})$ & $\mathrm{g}()$. \\
\hline$R S S I \leq-100$ & 0.125 & $-75>R S S I \geq-80$ & 0.625 \\
$-90>R S S I \geq-100$ & 0.25 & $-70>R S S I \geq-75$ & 0.75 \\
$-85>R S S I \geq-90$ & 0.375 & $-65>R S S I \geq-70$ & 0.875 \\
$-80>R S S I \geq-85$ & 0.5 & $R S S I \geq-65$ & 1 \\
\hline
\end{tabular}

is an available channel in the serving cell. While obtaining the RSSI value for the cell selection, the average of $N_{s}=5$ samples spread over at least $300 \mathrm{~ms}$ is taken in [15]. The flat fading channel is generated by Rayleigh distribution and the mobility is modelled by using Jakes' method. All users are randomly located in the cells.

The users are characterized with velocities up to the values given in Table 1. The mobile users can perform the cell reselection depending on their mobility and location in the cell. If their RSSI values are less than the given RSSI re-selection threshold during the last 10 measurements, the users detach from the serving cell and attach to the new one.

The sorted of RSSI values are obtained by using $f$ function in which measured RSSI values from all BSs are simply sorted and assigned to a normalized value according to Table 2. The normalized RSSI values are mapped using fixed intervals in $g$ function according to Table 3. Since the RSSI values are more intense in certain intervals, nonlinear mapping is applied to them.

All BSs calculate their UTL values at 1 second and then calculate the averaged value over the last 60 ones. Then, all BSs broadcast the corresponding index value at every second by using 2 bits as given in Table 4 . Since the calculation of
Table 5 Simulation parameters

\begin{tabular}{ll}
\hline Parameters & Tetra Trunk \\
\hline Defined time interval (D) & $1 \mathrm{~min}$ \\
Total simulation time & $30 \mathrm{~min}$ \\
Re-selection threshold & $-100 \mathrm{dBm}$ \\
Weight of RSSI $(w)$ & $0.2,0.5,0.8$ \\
Percentage of indoor users & $20 \%$ \\
Percentage of pedestrian users & $50 \%$ \\
Percentage of vehicle users & $30 \%$ \\
U threshold & 0.5 \\
c (initial cell selection) & 0 \\
c (cell re-selection) & 0.2 \\
\hline
\end{tabular}

UTL values is different for initial and cell re-selection cases, the mapping values are given for these two cases separately.

The performance metrics are the number of RSSI measurements and the number of requests while attaching to a $\mathrm{BS}$, and the average waiting time to establish communications. In addition to that, the outage probabilities in which the mobile user is not able to acquire the required BER in whole transmission time $\left(P_{\text {out }}=\operatorname{Pr}\left(B E R_{u, k}<B E R_{t h}\right)\right.$ where $\left.B E R_{t h}=0.025\right)$, are provided for all cell selection algorithms.

The simulation parameters for the proposed traffic aware system are listed in Table 5. The performance results are provided for urban, rural and suburban environments by choosing the frequency reuse factor is 3 . Then, the three frequencies which are $415 \mathrm{MHz}, 415.2 \mathrm{MHz}$ and $415.4 \mathrm{MHz}$ are assigned to the BSs.

For urban area, Hata path loss model [15]:

$$
\begin{aligned}
P L_{u, k}(d B)= & 69.55+26.16 \log _{10} f_{u}-13.82 \log _{10} h_{b} \\
& -\left(3.2\left(\log _{10}\left(11.75 h_{m}\right)\right)^{2}-4.97\right) \\
& +\left(44.9-6.55 \log _{10} h_{b}\right) \log _{10} R_{u, k}
\end{aligned}
$$

where $f_{u}$ is the frequency of BS u, $h_{b}$ represents BS antenna height, $h_{m}$ is mobile device antenna height and $R_{u, k}$ is the distance between BS $u$ and user $k$.
Table 4 Mapping traffic load values in $h$ function

\begin{tabular}{llll}
\hline Calculated UTL for initial selection & Averaged UTL for cell re-selection & Index & $h()$. \\
\hline $0-0.7$ & $0-0.6$ & 1 & 1 \\
$0.7-1.2$ & $0.6-1$ & 2 & 0.66 \\
$1.2-$ more & $1-$ more & 3 & 0.33 \\
None & No available channel & 4 & 0
\end{tabular}




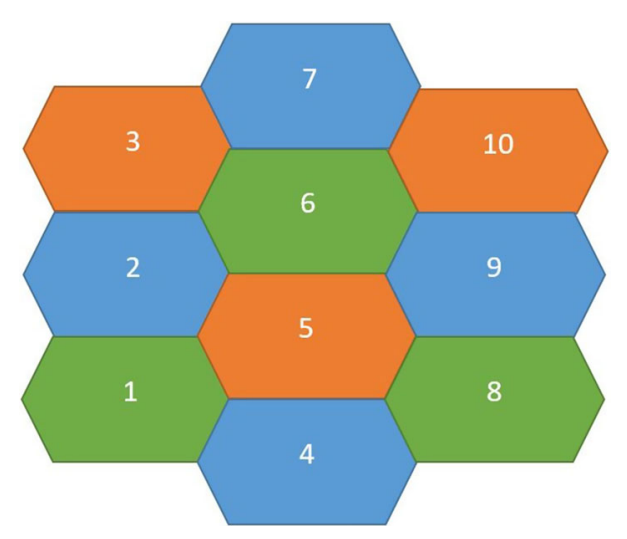

Fig. 3 Urban cell planning

For rural area, Hata path loss model [15]:

$$
\begin{aligned}
\mathrm{PL}_{\mathrm{u}, \mathrm{k}}(\mathrm{dB})= & 69.55+26.16 \log _{10} f_{u}-13.82 \log _{10} h_{b} \\
& -\left(1.1 \log _{10}\left(f_{u}\right)-0.7\right) h_{m} \\
& +\left(1.56 \log _{10}\left(f_{u}\right)-0.8\right) \\
& +\left(44.9-6.55 \log _{10} h_{b}\right) \log _{10} R_{u, k} \\
& -4.78 *\left(\log _{10}\left(f_{u}\right)\right)^{2} \\
& +18.33 * \log _{10}\left(f_{u}\right)-40.94
\end{aligned}
$$

For suburban area, Hata path loss model [15]:

$$
\begin{aligned}
P L_{u, k}(d B)= & 69.55+26.16 \log _{10} f_{u}-13.82 \log _{10} h_{b} \\
& +\left(44.9-6.55 \log _{10} h_{b}\right) \log _{10} R_{u, k}
\end{aligned}
$$

$$
-2\left(\log _{10}\left(f_{u} / 28\right)\right)^{2}-5.4
$$

The antenna height of BS and the mobile device are $30 \mathrm{~m}$ and $1.5 \mathrm{~m}$, respectively.

As shown in Fig. 3, in the urban environment, there are $10 \mathrm{BSs}$ and 500 users which are divided into 10 groups and PTT is chosen as 0.6. Therefore, there are 300 talking users in every minute. All BSs have 8 time slots (one for control purposes) which corresponds to 2 physical channels with 50 $\mathrm{kHz}$. Inter-site distances are chosen as $6.5 \mathrm{~km}$ for urban, $9 \mathrm{~km}$ for suburban and $28.5 \mathrm{~km}$ for rural environment.

The performance results for the urban environment are shown in Table 6. The proposed traffic aware algorithms give better performance than $\mathrm{C} 1$ algorithm in a manner of the average waiting time. While the proposed full set algorithm with $w=0.2$ reduces the waiting time about $20 \%$ than $\mathrm{C} 1$ algorithm, the proposed reduced set algorithm with $w=0.2$ reduces it about $15 \%$. The number of RSSI measurements is decreased around 55\% for the proposed traffic aware reduced set algorithm. With the increasing weight value in the proposed traffic reduced set algorithm, it is observed that the number of RSSI measurements is increased. The average number of requests is decreased about $30 \%$ for the proposed traffic aware algorithms. As given in Table 7, it is seen that the diversity has important effect on BER performances and the outage probabilities are below 0.05 , which is the required quality of service for Tetra Trunk PMR systems.

In the rural environment, the cell model is given in Fig. 4 including 5 BSs and 100 users with 3 groups. Only the BS at
Table 6 Performance results for the urban area
Table 7 Outage probabilities for the urban area

\begin{tabular}{llll}
\hline Algorithms results & Numberof RSSI measurements & Numberof requests & Average waiting time (s) \\
\hline $\mathrm{C} 1$ & 10 & 1.2115 & 2.5994 \\
Cell load in [11] & 10 & 1 & 2.3251 \\
Full set traffic $\mathrm{w}=0.2$ & 10 & 1 & 2.1247 \\
Full set traffic $\mathrm{w}=0.5$ & 10 & 1 & 2.1656 \\
Full set traffic $\mathrm{w}=0.8$ & 10 & 1 & 2.3148 \\
Reduced set traffic $\mathrm{w}=0.2$ & 3.049 & 1 & 2.3454 \\
Reduced set traffic $\mathrm{w}=0.5$ & 3.1811 & 1 & 2.2552 \\
Reduced set traffic $\mathrm{w}=0.8$ & 3.4713 & 1 & 2.3451 \\
\hline
\end{tabular}

\begin{tabular}{lll}
\hline Algorithms results & Outage probability without diversity & Outage probability with diversity \\
\hline $\mathrm{C} 1$ & 0.071454 & 0.027415 \\
Cell load in [11] & 0.0719 & 0.0295 \\
Full set traffic $\mathrm{w}=0.2$ & 0.0742 & 0.030763 \\
Full set traffic $\mathrm{w}=0.5$ & 0.0761 & 0.032031 \\
Full set traffic $\mathrm{w}=0.8$ & 0.07079 & 0.02884 \\
Reduced set traffic $\mathrm{w}=0.2$ & 0.0797 & 0.037013 \\
Reduced set traffic $\mathrm{w}=0.5$ & 0.08533 & 0.041533 \\
Reduced set traffic $\mathrm{w}=0.8$ & 0.08821 & 0.042558 \\
\hline
\end{tabular}




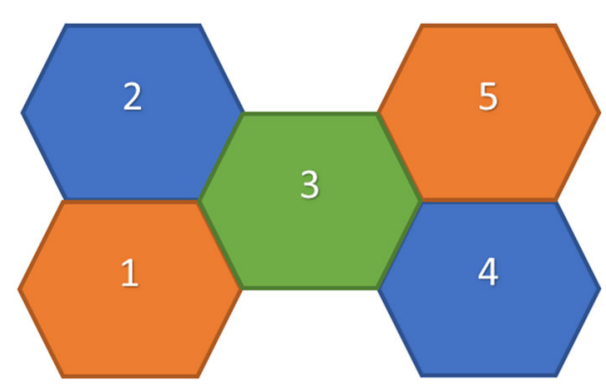

Fig. 4 Rural environment cell planning

the center of the area has a different frequency. The probability of PTT is 0.35 which results in 35 talking users in every minute. Since traffic volume is less in rural environments, 4 time slots (one channel for control channel) are assigned to each BS. This requires $25 \mathrm{kHz}$ bandwidth with 1 physical channel.

The performance results in the rural environment is given in Table 8. Due to the low traffic volume and low number of users, a significant reduction in the waiting time is observed compared to the urban environment. The proposed traffic aware algorithms with $\mathrm{w}=0.2$ give the best result in terms of the waiting time and reduces it about $40 \%$ than the $\mathrm{C} 1$ cell selection. The number of RSSI measurements is decreased about $70 \%$ and the number of requests is reduced about $25 \%$ in the proposed traffic aware reduced set algorithm. As shown in Table 9, the outage probabilities of all algorithms are below 0.05 .

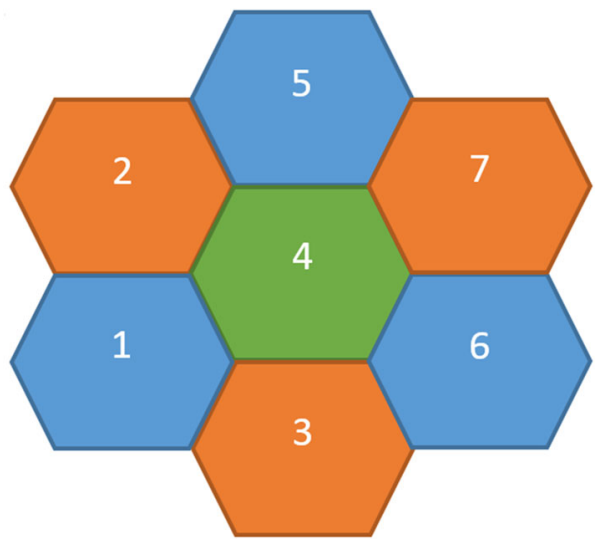

Fig. 5 Suburban environment cell planning

The cell modeling in suburban environment is given in Fig. 5. In this environment, the performance results are obtained with 300 users and 7 BSs. The users are divided into 3 groups and the probability of PTT is 0.20 . This results in 60 talking users in each minute and each BS has 4 time slots (one for control channel) which corresponds to $25 \mathrm{kHz}$ per BS.

As given in Table 10, in the suburban environemnt, it is observed around $45 \%$ reduction in the number of RSSI measurements for the proposed traffic aware reduced set algorithm. Since the users take into account the broadcasted traffic load values in the proposed algorithms, they can attach
Table 8 Performance results for the rural area

Table 9 Outage probabilities for the rural area

\begin{tabular}{llll}
\hline Algorithms results & $\begin{array}{l}\text { Number of RSSI } \\
\text { measurements }\end{array}$ & $\begin{array}{l}\text { Number of } \\
\text { requests }\end{array}$ & Average waiting time (s) \\
\hline C1 & 5 & 1.2493 & 0.53981 \\
Cell load in [11] & 5 & 1 & 0.48029 \\
Full set traffic w=0.2 & 5 & 1 & 0.46629 \\
Full set traffic w=0.5 & 5 & 1 & 0.46867 \\
Full set traffic w=0.8 & 5 & 1 & 0.48029 \\
Reduced set traffic w=0.2 & 4.4757 & 1 & 0.49067 \\
Reduced set traffic with w=0.5 & 4.5577 & 1 & 0.49533 \\
Reduced set traffic w=0.8 & 4.5838 & 1 & 0.49371 \\
\hline
\end{tabular}

\begin{tabular}{lll}
\hline Algorithms results & Outage probability without diversity & Outage probability with diversity \\
\hline $\mathrm{C} 1$ & 0.071 & 0.035 \\
Cell load in [11] & 0.062 & 0.027 \\
Full set traffic $\mathrm{w}=0.2$ & 0.067 & 0.035 \\
Full set traffic $\mathrm{w}=0.5$ & 0.064 & 0.029 \\
Full set traffic $\mathrm{w}=0.8$ & 0.064 & 0.027 \\
Reduced set traffic $\mathrm{w}=0.2$ & 0.059 & 0.026 \\
Reduced set traffic $\mathrm{w}=0.5$ & 0.061 & 0.027 \\
Reduced set traffic $\mathrm{w}=0.8$ & 0.064 & 0.0275
\end{tabular}


Table 10 Performance results for the suburban area

Table 11 Outage probabilities for the suburban area

\begin{tabular}{llll}
\hline Algorithms Results & $\begin{array}{l}\text { Number of RSSI } \\
\text { measurements }\end{array}$ & Number of requests & Average waiting time (s) \\
\hline $\mathrm{C} 1$ & 7 & 1.3013 & 2.7979 \\
Cell load in [11] & 7 & 1 & 2.5745 \\
Full set traffic $\mathrm{w}=0.2$ & 7 & 1 & 2.5173 \\
Full set traffic $\mathrm{w}=0.5$ & 7 & 1 & 2.5366 \\
Full set traffic $\mathrm{w}=0.8$ & 7 & 1 & 2.5772 \\
Reduced set traffic $\mathrm{w}=0.2$ & 4.1004 & 1 & 2.7034 \\
Reduced set traffic $\mathrm{w}=0.5$ & 4.2878 & 1 & 2.6743 \\
Reduced set traffic $\mathrm{w}=0.8$ & 4.3831 & 1 & 2.667 \\
\hline
\end{tabular}

\begin{tabular}{lll}
\hline Algorithms results & Outage probability without diversity & Outage probability with diversity \\
\hline $\mathrm{C} 1$ & 0.08112 & 0.010985 \\
Cell load in [11] & 0.0844 & 0.019332 \\
Full set traffic $\mathrm{w}=0.2$ & 0.0836 & 0.02768 \\
Full set traffic $\mathrm{w}=0.5$ & 0.0861 & 0.02351 \\
Full set traffic $\mathrm{w}=0.8$ & 0.0836 & 0.02017 \\
Reduced set traffic $\mathrm{w}=0.2$ & 0.0886 & 0.03018 \\
Reduced set traffic $\mathrm{w}=0.5$ & 0.0911 & 0.0402 \\
Reduced set traffic $\mathrm{w}=0.8$ & 0.0969 & 0.0377 \\
\hline
\end{tabular}

to the $\mathrm{BS}$ at their first attempt and the number of requests is decreased about $25 \%$ compared to $\mathrm{C} 1$ based cell selection algorithm. For the proposed traffic aware full set algorithm with $\mathrm{w}=0.2$, we provide about $15 \%$ decrease in the average waiting time than $\mathrm{C} 1$ algorithm. Again, from Table 11, outage probabilities of all algorithms are below 0.05 .

Besides, we compare the proposed traffic aware algorithm with the cell load based algorithm in [11] in which the utility function is determined based on the number of active users in the BSs and the RSSI values. Based on the performance results, the proposed traffic aware full set algorithm reduces the average waiting time about $10 \%$ further in all environments.

\section{Conclusion}

The cell selection plays a very critical role in Tetra Trunk based PMR systems. In this paper, we have proposed two cell selection algorithms and the performance of these algorithms have been provided by considering practical scenarios. The users can be mobile and the group talks are considered where the users have different duration of calls during the simulation time. In these proposed algorithms, the received power and traffic load of BSs have a weight in the utility function which decides the serving BS. While the RSSI values of all BSs is measured in the proposed traffic aware full set algorithm, the minimum number of RSSI measurements is targeted in the proposed traffic aware reduced set algorithm.

The performance results in different environments have been obtained. It has been shown that the fairly balancing of users among BSs reduces the average waiting time in the traffic aware cell selection algorithms. While the proposed traffic aware full set algorithm measures RSSI from all BSs, the proposed traffic aware reduced set algorithm measures much less RSSI values. Also decreasing RSSI weight has positive effect in balancing of the users and it also reduces the number of RSSI measurements. All these improvements in results have been occurred while satisfying the BER requirements of the users.

Acknowledgements This work has been supported by Republic Ministry of Science, Industry and Technology under SAN-TEZ 0686. STZ.2014 Programme.

\section{Compliance with ethical standards}

Conflicts of interest On behalf of all authors, the corresponding author states that there is no conflict of interest.

\section{References}

1. Gutierrez, F., \& Valdovinos, A. (1998). Performance improvement by using different transmission techniques for the digital 
mobile radio system TETRA. In Electrotechnical Conference, 1998. MELECON 98., 9th Mediterranean, Tel-Aviv, vol. 2, pp.726729.

2. Gao, Z., Chen, C., Li, Y., Wen, B., Huang, L., \& Zhao, Y. (2015). A mobility load balancing algorithm based on handover optimization in LTE network. In 2015 10th International Conference on Computer Science \& Education (ICCSE), Cambridge, pp. 611-614.

3. Park, W., Um, H., Ahn, J., \& Lee, S. (1997). Performance analysis on traffic load shedding schemes for mobile communication system. In Proceedings of ICUPC 97-6th International Conference on Universal Personal Communications, San Diego, CA, vol. 1, pp. 306-310.

4. Hanly, S. V. (1995). An algorithm for combined cell-site selection and power control to maximize cellular spread spectrum capacity. IEEE Journal on Selected Areas in Communications, 13(7), 13321340.

5. Akkarajitsakul, K., \& Phunchongharn, P. (2015). Joint cell selection and subchannel allocation for energy efficiency in small cell networks: A coalitional game. In 2015 International Conference on Wireless Communications \& Signal Processing (WCSP), Nanjing, pp. 1-6.

6. Qian, C., Zhang, S., Zhou, W. (2012). A novel cell selection strategy with load balancing for both idle and RRC-connected users in 3GPP LTE network. In 2012 International Conference on Wireless Communications and Signal Processing (WCSP), Huangshan, pp. $1-6$.

7. Moon, J. M., \& Cho, D. H. (2010). Cell Selection Algorithm Based on Competition of Users in Hierarchical Cellular Networks. 2010 IEEE Wireless Communication and Networking Conference, Sydney, Australia, pp. 1-6.

8. Nie, G., Tian, H., \& Ren, C. (2016). Energy efficient cell selection in small cell networks with constrained backhaul links. IEEE Communications Letters, 20(6), 1199-1202.

9. Thakur, R., Kotagi, V. J., \& Murthy, C. S. R. (2015). An energy efficient cell selection scheme for femtocell network with spreading. In 2015 IEEE 26th Annual International Symposium on Personal, Indoor, and Mobile Radio Communications (PIMRC), Hong Kong, pp. 1569-1573.

10. Tseng, P. H., Feng, K. T., \& Huang, C. H. (2014). POMDP-based cell selection schemes for wireless networks. IEEE Communications Letters, 18(5), 797-800.

11. Karatas, A., Ozbek, B., Sonmez, I., \& Bengur, S. (2016). Load based cell selection algorithm for Tetra based professional mobile radio. In 2016 24th Telecommunications Forum (TELFOR), Belgrade, pp. 1-4.

12. Terrestrial Trunked Radio (TETRA); Voice plus Data (V+D); Part 2: Air Interface (AI). ETSI TS 100 392-2 V3.6.1, 2013-05.

13. Karatas, A., Ozbek, B., Bardak, E. D., \& Sonmez, I. (2016). Cell selection algorithm performance for Tetra trunk. In 2016 24th Signal Processing and Communication Application Conference (SIU), Zonguldak, pp. 373-376.

14. Sangiamwong, J., Saito, Y., Miki, N., Abe, T., Nagata, S., \& Okumura, Y. (2011). Investigation on cell selection methods associated with inter-cell interference coordination in heterogeneous networks for LTE-advanced downlink. 17th European Wireless 2011-Sustainable Wireless Technologies, Vienna, Austria, pp. 16.

15. ETSI Technical Report143 030 V9.0.0 (2010-02), while the objective of the proposed traffic aware reduced set cell selection algorithm'Digital cellular telecommunications system (Phase 2+); Radio network planning aspects', 3GPP TR 43.030 version 9.0.0 Release 9, (2010).
Publisher's Note Springer Nature remains neutral with regard to jurisdictional claims in published maps and institutional affiliations.

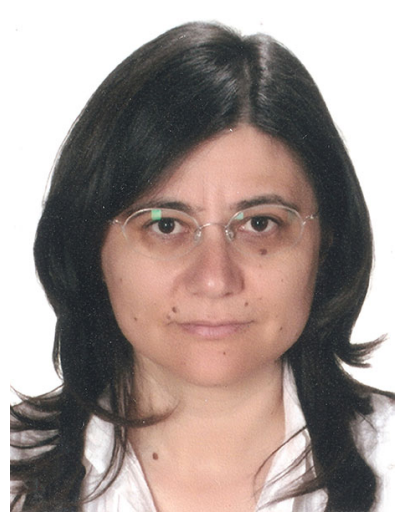

Berna Özbek is working in the position of an Associate Professor in Telecommunication field at the Electrical and Electronics Engineering Department of İzmir Institute of Technology, Turkey. She has graduated from Electrical and Electronics Department of Dokuz Eylul University, Turkey on 1994 and completed her MSc and $\mathrm{PhD}$ studies respectively on 1999 and 2004. Afterwards, she received a scholarship and worked as a postdoctoral researcher at CNAM-Paris on 2005. She was awarded as a Marie-Curie Intra-European (EIF) Fellow by European Commission for two years in the project entitled Interference Management Techniques for Multicell Networks on 2010. She has managed 1 international and 4 national projects, served as a consultant for 3 Eureka-Celtic projects and 2 national projects. Under her supervision, 11 master thesis and 2 doctoral dissertations have been completed. Currently, she is supervising $1 \mathrm{PhD}$ and 5 master students. She has published more than 80 peer-reviewed papers, 1 book, 1 book chapter and 2 patents. She is serving as a referee for several international journals, on numerous TPCs for IEEE sponsored conferences, European Commission, Turkish Republic of Ministry of Trade and Industry and The Scientific and Technological Research Council of Turkey. Her research interests are interference management, resource allocation and limited feedback strategies in multi-user, multi-antenna systems, device- to-device and heterogeneous wireless communications, physical layer security, massive MIMO systems, mmwave communications.

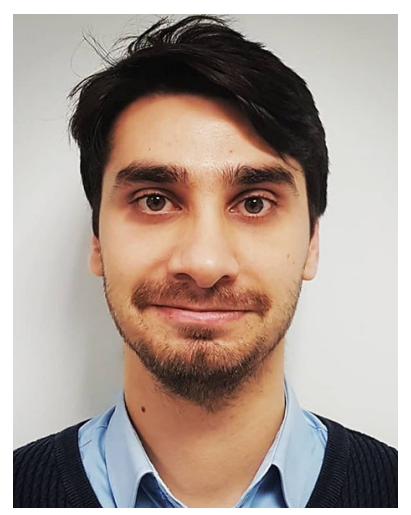

Azad Karataş has received his bachelor's degree and master's degree at Electronics and Communication Engineering in the Izmir Institute of Technology, respectively in 2014 and 2017. After he had worked in Izmir Institute Technology as a Research and Teaching Assistant for 3 years, he moved to The Scientific and Technological Research Council of Turkey in 2018 and working as an Electronics Engineer. His master's thesis was supported by Aselsan A.S. and the Ministry of Industry of Turkey for 2 years. He has published 2 international and 1 national conference papers. His research interests are mobile and satellite communication, software defined radios, embedded system design and machine and deep learning techniques. 


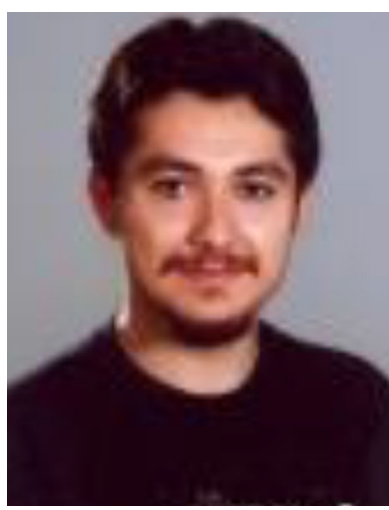

Erinç Deniz Bardak is an Electrical and Electronics engineer working as a systems engineer at ASELSAN Inc. for more than 10 years. $\mathrm{He}$ has graduated from Electrical and Electronics Department of Middle East Technical University, Turkey on 2006 and completed his MSc on 2009. He started working at ASELSAN Inc. in 2006 as a systems engineer in the field of communications, especially on public safety systems. He studied several public safety standards such as APCO25, TETRA and DMR. He worked in large scaled projects that are installed in several cities of Turkey for wide area communications. He is also working in projects about mission critical LTE and 5G solutions.

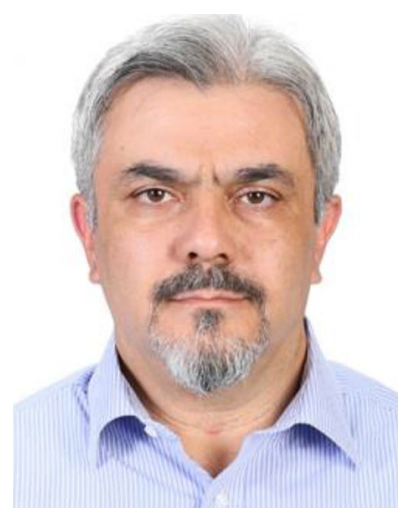

Ilker Sönmez is Lead Design Engineer in Aselsan Inc. He has took his undergraduate degree in 1994 from Hacettepe University Electric and Electronics Engineering Department. He has completed his Msc degree in the same university in 1997 . He has worked as a research assistant till the 1999 , in the same university in Electronics Engineering Department. Since 1999, he has been working as Telecommunication System Engineer in Aselsan Inc. He has more than 20 year experience in Digital Communication Systems, Digital Radio Networks, Architectural Design and Scaling of Radio Networks. 